\title{
LA RÉPARTITION DES WIEDEMANNIA DANS LES COURS D'EAU \\ ET LEUR UTILISATION COMME INDICATEURS DE ZONES ECOLOGIQUES \\ [Diptera, Empididae]
}

\author{
par F. Vaillant.
}

Les Wiedemannia sont des Diptères grêles, dont la taille reste comprise entre des limites étroites ${ }^{1}$ et dont la teinte est toujours grise, tirant sur le vert ou sur le brun.

Il $\mathbf{y}$ a, pour le moins, 43 espèces européennes, dont 23 sont représentées en France. Ces insectes présentent une grande uniformité, quant à leur habitat et à leur éthologie.

Les larves, à respiration aquatique, vivent exclusivement dans les eaux courantes, à l'intérieur des coussinets de mousse immergés à faible profondeur et recouvrant les pierres, parmi les algues filamenteuses ou exceptionnellement - W. phantasma (Mik) dans le sable en bordure des rivières; on en trouve aussi parfois à découvert dans des dépressions de la surface des pierres, ou dans des fissures. Elles se nourrissent presque uniquement de larves de Chironomidae, mais certaines - $W$. ouedorum VAILlaNT - peuvent également vivre aux dépens de larves de Simuliidae.

Les nymphes des Wiedemannia, nues et peu mobiles, ont le même habitat que les larves. Elles sont dépourvues de cornes respiratoires à plastron, de sorte qu'elles meurent rapidement une fois émergées. L'éclosion a généralement lieu dans l'eau et l'insecte, non mouillable, gagne la surface.

Les imagos ont un habitat assez restreint; il est limité presque exclusivement aux parties, mouillées par capillarité, des pierres des cours d'eau, juste au-dessus de la zone battue à intervalles réguliers par l'eau en mouvement. Elles restent à l'affût d'imagos d'Insectes qui émergent après leur éclosion ou bien qui viennent pondre à la limite de l'air et de l'eau. Leur nourriture est constituée presque totalement de Chironomidae, mais j'ai observé plusieurs Wiede-

1. Les plus grands mesurent en extension $7 \mathrm{~mm}$, alors que les plus petits ont au moins $5 \mathrm{~mm}$. 
mannia fallaciosa (LoEw) dépeçant une subimago d'Éphémère du genre Ecdyonurus; des Plécoptères sont aussi parfois consommés.

Lorsqu'un de ces Empidides est dérangé, il s'envole et se pose, non loin de là, sur une autre pierre, juste au-dessus de l'eau.

L'accouplement des Wiedemannia a lieu dans leur territoire de chasse et j'ai souvent vu des femelles déposant leurs œufs en ce même lieu.

La nuit, beaucoup d'individus mâles et femelles demeurent sur les pierres des cours d'eau.

Les $W$. beckeri Mrk, dont l'aire est limitée aux torrents de haute montagne, semblent univoltines; les larves âgées et les nymphes ne s'observent qu'en juillet et en août, en même temps que de très nombreuses imagos des deux sexes; fin septembre, on ne trouve plus que des femelles, dont l'abdomen est souvent distendu par les cufs. De jeunes larves passent par conséquent l'hiver dans les torrents. Toutefois, j'ai capturé quelques imagos — surtout des femelles - une quinzaine de jours après la fonte de la neige en juin; quelques individus resteraient donc vivants pendant l'hiver sous forme de larve âgée, ou de nymphe, ou d'imago. Les œufs de ces «imagos précoces» fourniraient les «larves tardives»du mois d'août.

Le cycle complet des Wiedemannia fallaciosa et des W. ouedorum, dans les régions basses et pendant la belle saison, est d'environ six semaines; ces espèces sont donc polyvoltines selon toutes probabilités, ainsi que les autres Wiedemannia de movenne et de faible altitude; on trouve des imagos de ces Diptères même en plein hiver.

Il n'y a pas lieu de s'étonner de ce que les collections de Diptères renferment en général peu de Wiedemannia. En effet il est tout à fait exceptionnel qu'un exemplaire soit capturé en fauchant avec un filet sur des plantes basses ou sur des arbres en bordure d'un cours d'eau.

Il vaut mieux ne pas employer cet instrument, si l'on cherche à capturer des Wiedemannia ${ }^{2}$ sur des pierres mouillées, car il serait vite trempé et inutilisable. D'autre part les Empidides de ce genre ne s'envolent, pour la plupart, qu'après le passage d'un filet audessus d'eux. Il est préférable de " coiffer », sur les pierres, les Wiedemannia, avec un flacon à cyanure, un par un ou groupe par groupe.

Ces insectes ne fréquentent que certaines pierres des cours d'eau, généralement les plus grosses, et certaines parties seulement de chaque pierre, de préférence les faces verticales ou en surplomb.

2. A moins qu'ils ne soient très nombreux. 


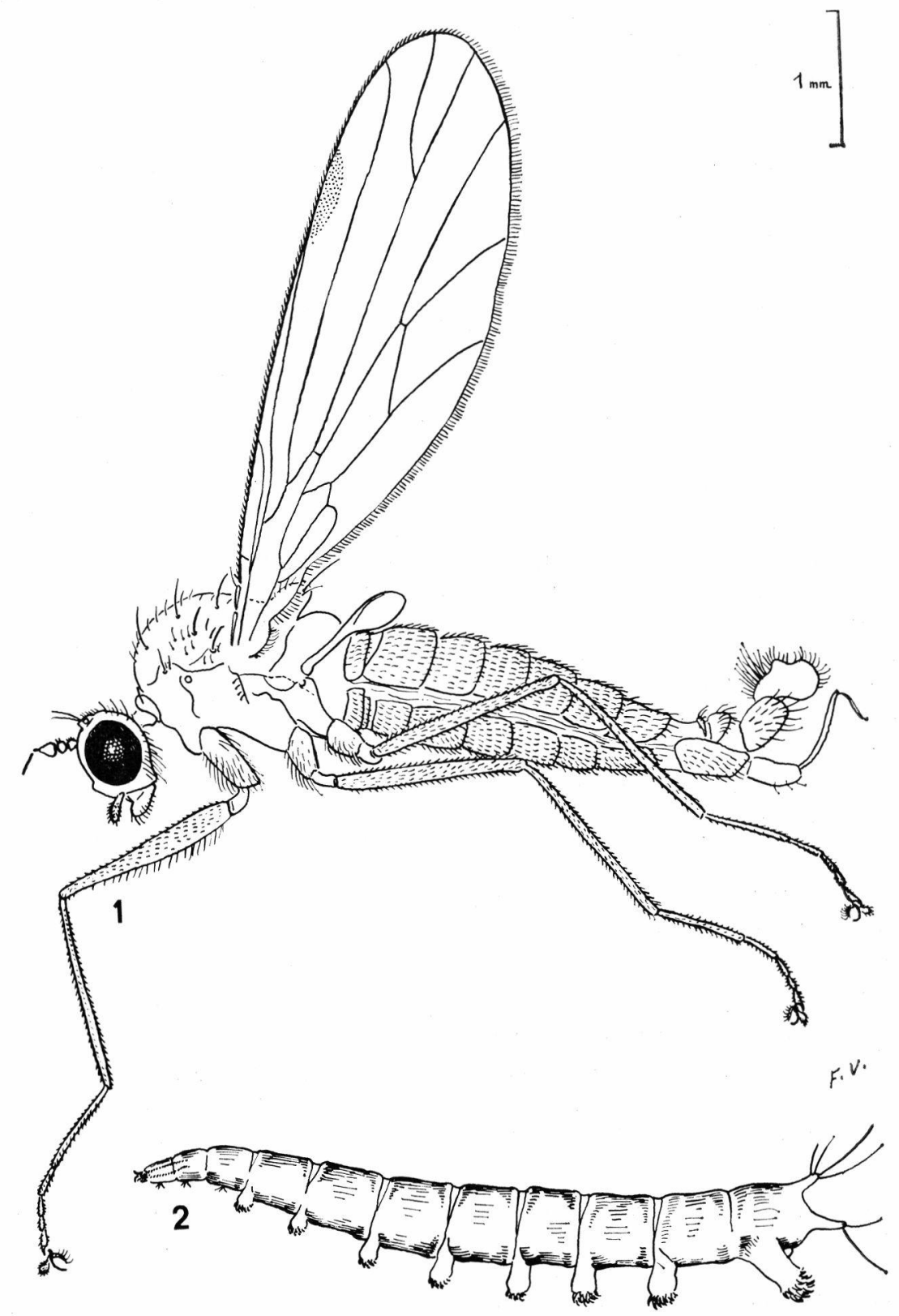

Planche 1 ,

FIG. 1 et 2 : Wiedemannia hygrobia Loew. -1 : imago $\hat{s}$ en extension; 2 : larve au dernier stade. 
Avec un peu d'habitude, on parvient à capturer, en un quart d'heure, plusieurs Wiedemannia - lorsqu'il y en a - dans n'importe quel cours d'eau et parfois, on en prend des centaines en quelques minutes.

Lorsqu'au bout d'une demi-heure, on n'a aperçu aucun exemplaire dans un torrent ou dans une rivière, point n'est besoin d'insister. Plusieurs facteurs peuvent entraîner l'absence de ces Diptères et je reviendrai sur cette question page 289 .

M'étant aperçu que des espèces de Wiedemannia se succédaient dans certains cours d'eau, de l'amont vers l'aval, j'ai trouvé intéressant de chercher à apporter quelques précisions sur cette question et de comparer les séries dans plusieurs rivières du bassin de la Garonne, de celui du Rhône, et de celui de la Loire.

Plusieurs hydrobiologistes ont tenté d'établir une zonation des cours d'eau, en utilisant comme indicateurs des Poissons, des Turbellariés Triclades, des groupes d'Invertébrés. Dans le mémoire de J. Illies et L. Botosaneanu, une excellente révision de ces travaux a été donnée et une nouvelle zonation a été proposée. J'ai essayé de voir si les Wiedemannia, accompagnant presque partout les eaux courantes, étaient aussi de bons indicateurs de zones.

Il eut paru préférable de s'adresser aux larves, aquatiques, qu'aux imagos, aériennes. Plusieurs raisons m'ont conduit à choisir ces dernières.

Tout d'abord les larves sont dispersées sur les pierres des cours d'eau et n'ont pas, comme les imagos, de petits territoires d'élection; aussi sont-elles fort difficiles à découvrir.

D'autre part il est illusoire de vouloir les identifier. En effet, au cours de ces dernières années, j'ai réussi, à l'aide d'élevages, à déterminer les larves de plusieurs espèces de Wiedemannia; mais je n'ai pu découvrir aucun caractère taxonomique permettant de les séparer; toutes répondent à la description que j'ai donnée en 1951 de la larve de $W$. ouedorum.

Enfin, j'ai pu constater, chaque fois que j'ai découvert des larves et réussi à les élever, que des imagos de la même espèce se trouvaient, sur les pierres, dans la portion du cours d'eau qui hébergeait les larves. Il semble donc que les imagos des Wiedemannia ne changent pas souvent de territoire après leur éclosion et qu'on puisse utiliser celles-ci au même titre que les larves. 
Cette étude m'a conduit à découvrir quatre espèces nouvelles de Wiedemannia et à distinguer trois sous-espèces chez W. beckeri. Je les décrirai avant d'aborder les deux questions qui font l'objet essentiel de ce travail.

La nomenclature utilisée pour la nervation aiaire est celle de O. ENGEL et, pour l'armure génitale, celle de R. BährmanN.

Wiedemannia (Chamaedipsia) aerea n. sp. (fig. 2.1 et 2.2). Mâle : Joue haute comme un tiers de la hauteur de l'œil. Face profondément échancrée et saillante en museau à la partie supérieure de l'échancrure; la saillie est bordée de deux crêtes convergentes vers le haut. Face à poudré gris bleu, légèrement brune sur le pourtour de l'échancrure et sur les côtés. Front et ocriput à poudré gris bleu, mais il y a un triangle médian brun foncé sur le front et le mamelon ocellaire a la même couleur. Occiput à poudré gris verdâtre, légèrement rembruni dans sa partie médiane. Deux chètes interocellaires, 8 ou 9 soies postocellaires; de chaque côté 8 ou 9 chètes postoculaires supérieurs, dont le quatrième est beaucoup plus long que les autres; de nombreux chètes bien développés entre eux et le cou. De courtes soies claires éparses en arrière des yeux et en dessous du niveau du cou. Mesonotum brun foncé avec une bande brun clair entre les deux rangées de dorso centraux et une étroite bande gris jaunâtre, sur la ligne médiane, s'arrêtant avant la dépression préscutellaire. Côtés du mésonotum, triangle sutural, dépression préscutellaire, scutellum, mésophragme et pleurites à poudré gris bleu foncé. Sept ou huit acrosticaux irrégulièrement alignés sur la moitié antérieure du mésonotum et trois paires dans la dépression préscutellaire; de chaque côté, $8 \mathrm{dc}$, avec des chètes intercalaires bien développés, 1 chète et 1 chétule huméraux, 1 chète présutural, 1 chète et 6 chétules dans le triangle sutural, 1 grand chète supraalaire et 10 à 12 chètes entre chaque rangée de dc et la suture notopleurale correspondante. Scutellum : 2 chètes et 4 chétules marginaux et au moins 4 chétules sur le disque. Hanche à poudré gris bleu foncé; pattes brunes. Fémur I : 1 chète antérieur, au 1/4 distal, et un autre, antérieur également et préapical; ventralement, des soies, dont certaines sont presque aussi longues que le travers du fémur. Fémur II et III : $\mathbf{2}$ chètes préapicaux antérieurs un peu plus longs que les autres. Aile à tache stigmatique peu nette, large comme les $2 / 3$ de la cellule radiale; la cellule discale et la deuxième cellule postérieure ne sont pas contiguës, mais réunies par un segment de la nervure $\mathrm{m} 1 \mathrm{~m} 2$, parfois aussi long que la traverse mcu; cellule anale arrondie à son extrémité et nervure anale à peine amorcée.

Longueur du corps : $5,5 \mathrm{~mm}$. Longueur de l'aile : 4,2 à $4,5 \mathrm{~mm}$.

Femelle : mêmes caractères que ceux du mâle. 


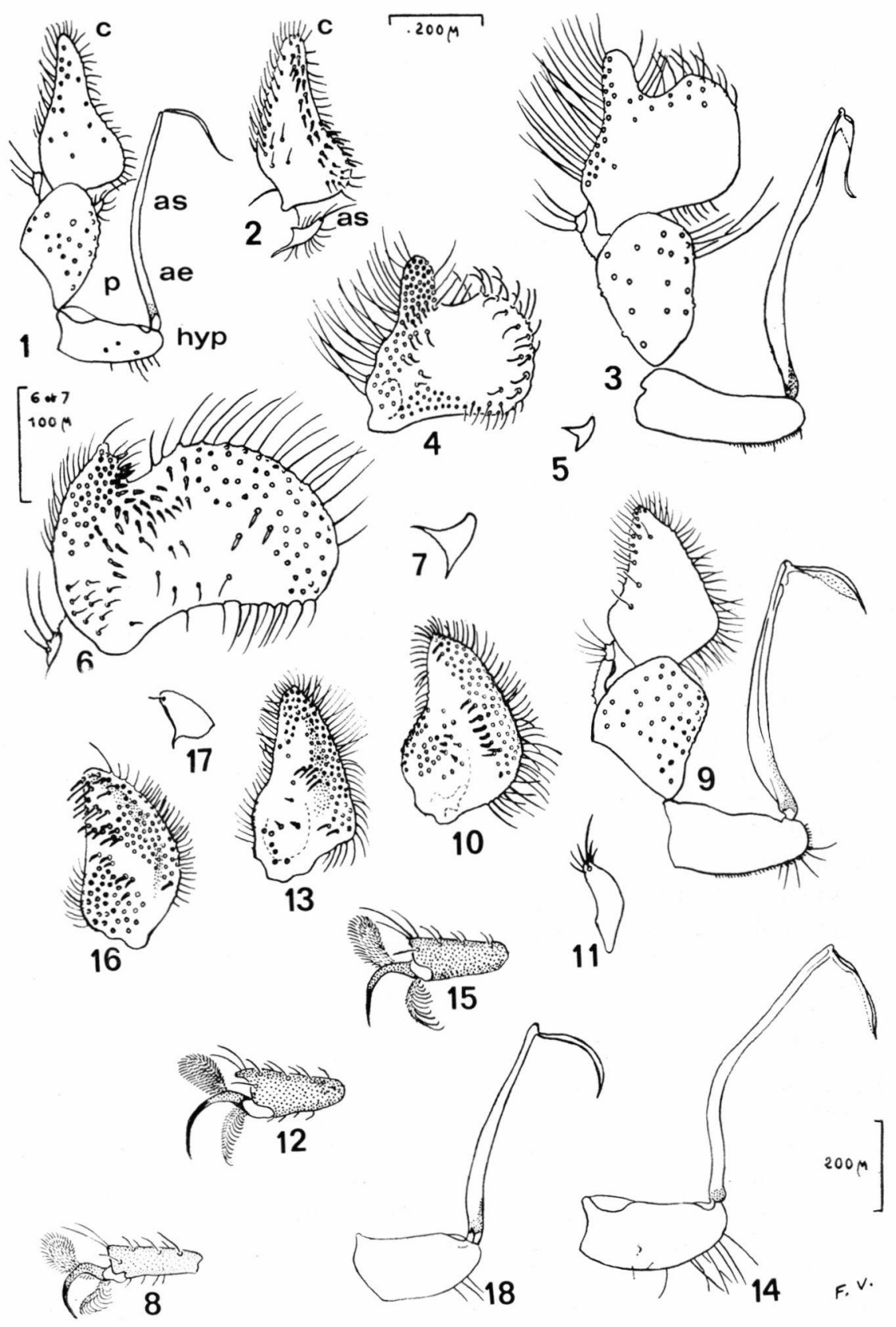

Planche 2.

Fig. 1 et 2 : Wiedemannia aerea n. sp. $\hat{\delta}$ - 1 : genitalia, profil; 2 : cerque et appendice secondaire droit, face interne.

FIg. 3 à 5 : Wiedemannia angelieri n. sp. $ఠ .-3$ : genitalia, profil; 4 : cerque droit, face interne; 5 : appendice secondaire droit, face interne.

Fig. 6 et 7 : Wiedemannia queyrasiana VaIllant $\hat{\delta} \cdot-6$ : cerque droit, face interne; 7 : appendice secondaire droit, face interne.

FIG. 8 à 11 : Wiedemannia beckeri alpina n. ssp. $\hat{\text {. }}$ - 8 : dernier article du tarse II droit, profil; 9 : genitalia, profil; 10 : cerque droit, face interne; 11 : appendice secondaire droit, face interne.

Fıg. 12 à 14 : Wiedemannia beckeri pyrenaica n. ssp. §ิ. - 12 : dernier article du tarse II droit, profil; 13 : cerque droit, face interne; 14 : hypandrium et aedeagus, profil.

Fig. 15 à 18 : Wiedemannia beckeri carpathica n. ssp. $\hat{\text { s. }}-15$ : dernier article du tarse II droit, profil; 16 : cerque droit, face interne; 17 : appendice secondaire droit, face interne; 18 : hypandrium et aedeagus, profil.

ae : aedeagus; - as : appendice secondaire; - c : cerque; - p : epandrium; - hyp : hypandrium.

Toutes les figures sont à la même échelle, sauf les figures 6 et 7 . 
Types capturés le 15-IX-1966 dans le torrent de la Roche Noire (Hautes-Alpes), à $2050 \mathrm{~m}$ d'altitude.

W. (Ch.) aerea se rapproche beaucoup de W. (Ch.) mikiana BEzzi, des Alpes italiennes et autrichiennes, trouvé également en Hongrie, mais il s'en sépare nettement par la forme des cerques chez le mâle.

Wiedemannia (Philolutra) angelieri n. sp. (fig. 2.3 à 2.5 et 3.1 ). Mâle : Joue haute comme un quart de la hauteur de l'œil. Face profondément échancrée et saillante en museau, comme chez l'espèce précédente. Face à poudré gris bleu foncé, rembrunie sur le pourtour de l'échancrure. Front et toute la face postérieure de la tête brun noir, avec un léger poudré gris en dessous du niveau du cou. Deux chètes interocellaires et 8 à 10 soles postocellaires; de chaque côté, 4 chètes postoculaires supérieurs de même longueur et fort éloignés de l'œil; entre celui-ci et la rangée de postoculaires sont de nombreux chétules. Sur toute la face postérieure de la tête, en arrière des postoculaires supérieurs, il y a de longues soies claires et il s'en trouve d'autres en dessous du niveau du cou. Pronotum, mésonotum, scutellum, mésophragme et liseré supérieur des pleurites en grande partie à poudré gris bleu. Pronotum : 4 chétules. Mésonotum : 24 à 26 chétules acrosticaux bisériés; de chaque côté, 5 à $6 \mathrm{dc}$, avec de rares intercalaires de très petite taille. 1 seul chète huméral, 1 chète et 3 ou 4 chétules présuturaux, 2 chètes et quelques soies très fines dans le triangle sutural et 1 supraalaire. Scutellum : 2 chètes et 4 à 6 soies marginaux, 2 soies sur le disque. Hanche à poudré gris bleu et à soies roussâtres. Fémur I : une rangée antéro-ventrale et une rangée postéro-ventrale de soies. Tibia III : quelques chètes particulièrement forts sur le 1/3 distal, dont 2 ou 3 postéro-ventraux, 4 antéro-ventraux et 10 à 12 dorsaux. Aile sombre, à tache costale nette et large comme les $3 / 4$ de la cellule radiale; la transverse $\mathrm{rm}$ et la transverse qui représente le premier segment de m2 sont généralement de même longueur. La cellule anale est arrondie à son extrémité et la nervure anale, nette et allongée, part très en avant de l'extrémité de la cellule. Les cerques sont recourbés en crocheí dans leur partie postérieure, comme le montre la figure 3.1 .

Longueur : 5,6 mm. Longueur de l'aile : 4,3 à $4,5 \mathrm{~mm}$.

Femelle : mêmes caractères que ceux du mâle.

Types capturés, le 28-VIIJ-1967 au col de Puymorens (Pyrénées(irientales), vers $1920 \mathrm{~m}$.

Cette espèce, que j'ai le plaisir de dédier à mon collègue, M. E. Angelier, se rapproche d'une autre Philolutra des Alpes, beaucoup plus petite, $W$. (Ph.) queyrasiana Valllant; elle s'en distingue nettement toutefois par la présence d'une transverse, à la limite 
entre cellule discale et deuxième cellule postérieure, par l'absence, presque régulière, d'un chète préapical antérieur sur le fémur I, et par la forme et l'ornementa'ion des cerques chez le mâle. Il m'a paru préférable de figurer (fig. 2.6) à nouveau, à titre comparatif, un cerque de $W$. (Ph.) queyrasiana.

Wiedemannia (Chamaedipsia) beckeri Mrk.

En 1964, j'avais signalé cette espèce des Pyrénées. Un examen plus poussé m'a montré que le $W$. beckeri des Alpes différait de celui des Pyrénées par de nombreux caractères; il y a donc lieu de distinguer 2 sous-espèces.

L'hydrobiologiste roumain, M. L. Botosaneand m'a envoyé des W. beckeri capturés par lui dans les Carpathes et qui appartiennent à une troisième sous-espèce.

Les $W$. beckeri des Alpes, ceux des Pyrénées et ceux des Carpathes ont la même taille et à peu près la même coloration. Voici quelques caractères permettant de les distinguer :

W. beckeri alpina $\mathrm{n}$. ssp. (fig. 2.8 à 2.11). - Téguments moyennement pigmentés. Dernier article de chaque tarse avec un prolongement dorsal peu marqué. Chez le mâle, cerques de forme grossièrement losangique et zone couverte d'épines réduite à une bande sur le bord postérieur; appendices secondaires (Sekundäre Abgliederungen) bien développés, avec de longues soies terminales; hypandrium allongé et aedeagus renflé à sa base. Les spécimens de référence ont été capturés en juillet et août 1966 dans le Rif du Col au Lautaret (Hautes-Alpes) entre 2050 et $2070 \mathrm{~m}$ d'altitude.

W. beckeri pyrenaica n. ssp. (fig. 2.12 à 2.14). - Téguments très pigmentés. Dernier article de chaque tarse avec un prolongement dorsal très saillant. Chez le mâle, cerques de forme grossièrement triangulaire et zone couverte d'épines réduite à une bande sur le bord postérieur; appendices secondaires réduits et sans soies; hypandrium plus court que chez la sous-espèce précédente; aedeagus d'épaisseur régulière. Types capturés le 2-VII-1957 par M. R. MInouse au Col de Puymorens (Pyrénées Orientales).

W. beckeri capathica n. ssp. (fig. 2.15 à 2.18). -- Téguments peu pigmentés. Dernier article de chaque tarse avec un prolongement dorsal un peu saillant. Chez le mâle, cerques de forme grossièrement semi-circulaire et zone couverte d'épines très étendue; appendices secondaires courts, avec une ou deux soies minuscules; hypandrium encore plus court que chez $W$. beckeri pyrenaica et aedeagus légèrement renflé à sa base.

Types : Le Zapada, in Turul 'Lacului Capra (Carpathes de Roumanie) coll. L. BotosaneanU. 


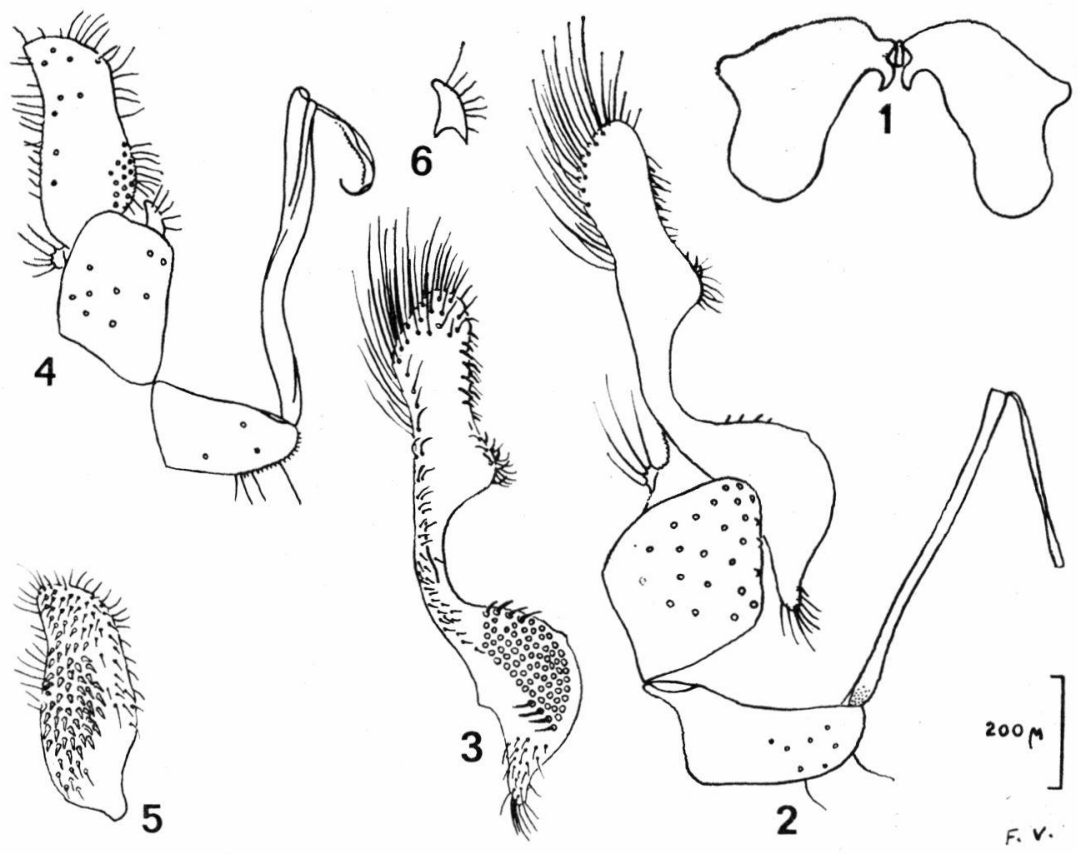

Planche 3.

Fıg. 1 : Wiedemannia angelieri n. sp. 1 , cerques étalés et vus par leur face postérieure.

Fig. 2 et 3 : Wiedemannia falcifera n. sp. $1 .-2$ : genitalia, profil; 3 : cerque droit, face interne.

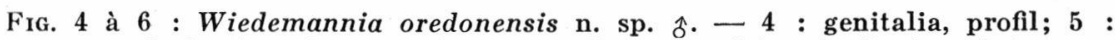
cerque droit, face interne; 6 : appendice secondaire droit, face interne.

Wiedemannia (Wiedemannia) falcifera n. sp. (fig. 3.2 et 3.3).

Mâle : Joue haute comme les $3 / 5$ de la hauteur d'un œil. Face échancrée, le sommet de l'échancrure se trouvant un peu en dessous du niveau du bord inférieur des yeux; celle-ci est située sur une saillie et bordée de deux facettes. La face est gris bleu, mais l'échancrure, ainsi que les bords des facettes, sont soulignés par une bande brune. Le front, le mamelon ocellaire et la plus grande partie de l'occiput sont bruns; les parties latérales de l'occiput et la face postérieure de la tête en dessous du cou sont gris bleu. Deux interocellaires et 8 postocellaires. De chaque côté, 7 grands postoculaires noirs mal alignés et de nombreux chétules entre leur rangée et l'œil correspondant, ainsi qu'entre elle et le cou. En dessous du niveau du cou, de grandes soies claires peu nombreuses. Pronotum, mesonotum, scutellum, mésophragme et une large bande en dessous 
de chaque suture notopleurale brun foncé unitorme; pleures en majeure partie gris bleu. Pronotum : 2 chètes. Mesonotum : plus de 30 chétules acrosticaux bisériés, en avant de la dépression notopleurale, et irrégulièrement tétrasériés sur celle-ci. De chaque côté, 6 dc, sans intercalaires, 2 chètes et 3 ou 4 chétules huméraux, 1 chète et de nombreux chétules présuturaux, 2 chètes et plusieurs chétules dans le triangle sutural, 1 chète supraalaire. Scutellum : 2 chètes et 6 à 8 soies marginaux; 8 à 12 soies sur le disque. Hanches gris bleu à soies claires; pattes brun noir. Fémur I : 1 chète préapical antérieur. Tibia III : quelques soies ventrales assez fortes sur la $1 / 2$ distale. Aile : nervure $r 2+3$ très incurvee au niveau de la tache stigmatique, assez pâle, presque circulaire et qui s'étend sur la moitié de la première cellule sous-marginale. Une droite, perpendiculaire à la nervure $\mathrm{r} 2+3$ au niveau de sa fourche, formerait un axe de symétrie pour la tache stigmatique. Le premier segment de $\mathrm{m} 2$ est très court. La cellule anale est arrondie à son extrémité et la nervure anale, distincte, est soulignée par une tache brune de la membrane.

Longueur du corps : 7,0 mm. Longueur de l'aile : 5,0-5,2 $\mathrm{mm}$.

Femelles : Mêmes caractéristiques que celles du mâle, mais la nervure $\mathrm{r} 2+3$ est moins incurvée et la tache stigmatique est à peine visible en dessous de celle-ci.

Types : L'Ariège, en amont de Merens (Ariège), $1060 \mathrm{~m}$, 28-VIII-1967.

Cette espèce est très voisine de $W$. (W.) bilobata Otdengerg, connue d'Engadine et de Carinthie; elle en diffère par plusieurs caractères, notamment par la présence de 2 chètes huméraux, au lieu de 3 ou 4, par l'absence d'intercalaires entre les chètes dorsocentraux, par la faible pigmentation de la tache stigmatique et par la forme un peu différente des cerques chez le mâle. Il n'en reste pas moins vrai que l'on pourrait considérer $W$. (W.) falcifera comme une simple sous-espèce pyrénéenne de $W$. (W.) bilobata.

Wiedemannia (Chamaedipsia) oredonensis n. sp. (fig. 3.4 à 3.6).

Mâle : Joue haute comme le $1 / 4$ de la hauteur d'un œil. Chétosité de la tête comme chez W. (Ch.) aerea (voir p. 271). Pronotum : 2 chètes puissants. Mesonotum : 22 à 26 chétules acrosticaux confusément bisériés et s'étendant sur la dépression préscutellaire. De chaque côté, $5 \mathrm{dc}$, avec plusieurs chétules en avant de la rangée, et 1 ou 2 intercalaires, 1 chète et 2 chétules huméraux, 1 chète et 4 ou 5 forts chétules présuturaux, 1 chète et 2 chétules dans le triangle sutural, 1 chète et 1 chétule supraalaires. Scutellum : seulement 2 chètes et 2 chétules marginaux. Fémur I : 2 chètes antérieurs alignés longitudinalement au $1 / 4$ distal et 1 chète pré- 
apical antérieur. Les fémurs II et III ont plusieurs chètes apicaux assez longs, mais nettement plus courts que le travers du segment. Tibia III : 3 ou 4 chètes dorsaux, plus forts que les autres, sur le $1 / 4$ distal. Le premier article de chaque tarse a, à sa base, 1 fort chète ventral. Aile uniformément rembrunie; sa tache costale, à peine visible, est large comme les $2 / 3$ de la celluie radiale; premier segment de $\mathrm{m} 1$ court ou absent; cellule anale arrondie à son extrémité et nervure anale marquée seulement par une ombre. Les genitalia sont ici fort particuliers, mais rappellent un peu toutefois ceux de W. (Ch.) longicornis Mrk.

Longueur du corps : $5,5 \mathrm{~mm}$. Longueur de l'aile : $4,5 \mathrm{~mm}$.

Un seul mâle a été capturé, le 31-VIII-1967, tout près du lac d'Orédon (Hautes-Pyrénées), dans le torrent de l'Estaragne, et vers $1950 \mathrm{~m}$.

$W$. (Ch.) oredonensis se distingue de W. (Ch.) longicornis Мıк en ce que son scutellum est inerme sur le disque.

$W$. (Ch.) oredonensis, comme W. (Ch.) beckeri Mı et W. (Ch.) alpina ENGEL, a un scutellum inerme sur le disque, des chètes acrosticaux nombreux, qui s'étendent jusqu'en arrière du scutellum; mais cette nouvelle espèce se sépare de $W$. (Ch.) beckeri en ce que la tache costale de son aile est peu visible et de $W$. (Ch.) alpina en ce que les 2 chètes antérieurs non apicaux du fémur I sont proches l'un de l'autre.

J'ai choisi, pour cette étude, sept cours d'eau.

Deux d'entre eux sont dans le bassin de la Garonne; ce sont l'Ariège, qui prend sa source dans les Pyrénées, et le Tarn, qui naît dans le Massif Central; l'un et l'autre coulent tout d'abord sur des granits, puis sur des roches calcaires.

Quatre des cours d'eau sont dans le bassin du Rhône; tous descendent des Alpes. Le torrent du Lautaret avec la portion aval de la Romanche, ainsi que la Durance, naissent dans des massifs cristallins, mais ont creusé leur lit dans des roches sédimentaires; le Furon et la Vence ne coulent que sur des roches calcaires. J'ai étudié, mais fort incomplètement, d'autres cours d'eau, affluents du Rhône et descendant du Massif Central.

Enfin la Loire a été explorée dans sa portion amont.

L'A riège et la Garonne. — L'Ariège naît, vers $2300 \mathrm{~m}$ d'altitude, de nombreuses sources rhéocrènes coulant parmi des blocs de granit et des Sphagnum. La pente est très douce. Elle s'accentue ensuite et les ruisselets confluent. Certaines pierres de l'unique cours d'eau sont recouvertes de mousse; celui-ci devient plus rapide et traverse un étang, le Lacul Negru et, plus bas, reçoit plusieurs petits 
affluents. L'Ariège coule ensuite dans une vallée encaissée; son lit est encombré de gros blocs cristallins, et sa pente est rapide. Après Mérens-les-Vals $(1050 \mathrm{~m})$, les rives, escarpées, sont formées de calcaires, mais le torrent continue à couler surtout sur des blocs cristallins. Après Ax-les-Thermes $(718 \mathrm{~m})$, la pente diminue et les pierres du torrent deviennent beaucoup plus pctites. A Tarascon $(474 \mathrm{~m})$, le faciès du torrent n'est pas très différent, mais l'eau commence à être polluée; elle l'est encore davantage à Saint-Jeande-Verges $(350 \mathrm{~m})$. A Pinsaguel $(155 \mathrm{~m})$, le lit de l'Ariège, tout comme celui de la Garonne dans laquelle elle se jette, est formé d'une dalle de calcaires coquillers creusée de sillons longitudinaux.

Dans l'Ariège on observe une remarquable succession de sept espèces de Wiedemannia. Je n'ai pu apercevoir aucun specimen entre les sources et le ruisseau à l'altitude $2200 \mathrm{~m}$, où les $W$. angelieri étaient seules représentées. A $2150 \mathrm{~m}$, les W. hygrobia remplaçaient l'espèce précédente et elles étaient encore plus abondantes à $1600 \mathrm{~m}$, mais mêlées à des $W$. bohemani. Plus bas, en amont de Mérens et vers $1050 \mathrm{~m}$, aucune des trois espèces précédentes n'était représentée, mais j'ai capturé de nombreux $W$. fallaciosa et quelques $W$. falcifera. En amont d'Ax-les-Thermes, à $720 \mathrm{~m}$, les seules Wiedemannia étaient des W. ouedorum et des W. rhynchops mirousei peu nombreuses. On retrouvait des exemplaires de cette dernière espèce, seuls et abondants, à Tarascon $(474 \mathrm{~m})$. Aux $W$. rhynchops mirousei se mêlaient, à Saint-Jean-de-Verges $(350 \mathrm{~m})$, quelques $W$. bistigma. Sur les arêtes, qui séparent les sillons en bordure des lits de l'Ariège et de la Garonne, à Pinsaguel $(155 \mathrm{~m})$, avant la confluence des deux rivières, j'ai pu capturer quelques W. bistigma.

En remontant la Garonne, un peu en aval de Labroquère, à $440 \mathrm{~m}$, celle-ci a un lit de cailloutis et j'y ai capturé des $W$. rynchops mirousei, ainsi que des $W$. bistigma, par conséquent les mêmes Diptères qu'à Saint-Jean-de-Verges.

Le Tarn et la Dourbie. - Les sources du Tarn sont peu différentes de celles de l'Ariège, bien qu'elles soient siluées vers $1500 \mathrm{~m}$, donc à une altitude plus faible. Elles apparaissent, sur le Mont Lozère, dans des prairies à tourbe, parmi les Sphagnum et les blocs de granit. Des ruisselets alternent avec de petits bassins. En amont de la route qui relie l'Hôpital à Bellecoste, la pente s'accentue et le Tarn s'engage plus bas dans une vallée encaisseje; le lit est formé de dalles granitiques creusées de "pots d'ogre ». Après Cocures, la pente diminue et le Tarn coule sur des blocs cristallins et des galets. Le Tarnon, dont les eaux sont très polluées à Florac, se jette dans le Tarn un peu en aval de cette ville. Puis la rivière pénètre dans les Causses; son lit devient calcaire et son cours de plus en 
plus lent. De profonds bassins presque stagnants alternent avec de courts tronçons où la pente est plus forte et où des pierres émergent; c'est là que se localisent les Wiedemannia.

Sur près de deux kilomètres en aval de la source du Tarn, la rivière, fort petite, semble dépourvue de Wiedemannia; mais peu avant la route de l'Hôpital à Bellecoste et vers $1300 \mathrm{~m}$, les $W$. ouedorum apparaissent subitement. A Pont-de-Monvert $(875 \mathrm{~m})$, elles sont remplacées par des $W$. bohemani et des W. zetterstedti. A Pont-du-Tarn $(540 \mathrm{~m})$, on trouve l'association $W$. zetterstedti - W. bifida et, à Sainte-Enimie $(470 \mathrm{~m})$, celle $W$. bificia - W. bistigma. Enfin, à Les Vignes $(420 \mathrm{~m})$, ainsi qu'à Millau $(379 \mathrm{~m})$, les W. bistigma sont seules représentées. Mais, dans la Dourbie, qui se jette dans le Tarn à Millau, on retrouve l'association W. zetterstedti - W. bifida observée à $540 \mathrm{~m}$ dans la rivière principale. En aval de Millau, le Tarn devient trop lent pour que les Wiedemannia puissent s'y développer.

Le torrent du Lautaret, la Romanche et l'Isère. - Le torrent du Lautaret provient en majeure partie du « Rif du Col ». Celui-ci nait d'une source rhéocrène sur les flancs du Combeynot à $2074 \mathrm{~m}$ d'altitude, à moins de $500 \mathrm{~m}$ des sources de la Guisanne, torrent qui descend vers Briançon. Le Rif du Col dévale rapidement sur des blocs de granit et sur des cailloutis; son eau ne contient que des traces de Ca et de matières organiques dissoutes; le cours d'eau s'infléchit vers $2050 \mathrm{~m}$ d'altitude et coule en pente douce sur des pierres, dont les unes sont granitiques, mais dont d'autres sont formées de schistes et de calcaires; le Rif du Col, devenu torrent du Lautaret, reçoit des affluents venus du massif granitique du Combeynot et d'autres, plus importants, qui proviennent de la Montagne de Chaillol; celle-ci est formée de calcschistes, de sorte que l'eau du torrent s'enrichit en $\mathrm{Ca}$ et devient légèrement séléniteuse et ferrugineuse.

La source du Rif du Col tarit, certaines années, au mois de septembre, mais de l'eau continue à couler sous les cailloutis, et les affluents venus de la Montagne de Chaillol maintiennent leur activité; le torrent du Lautaret n'est donc lui-même jamais à sec et d'importants coussinets de mousse (Cratoneuron glaucum) recouvrent certaines pierres.

La pente du torrent augmente vers $1900 \mathrm{~m}$, après la combe Girardin, et reste forte jusqu'à Arsine. Le cours d'eau atteint la Romanche à $1662 \mathrm{~m}$.

Les Empidides du cours supérieur de la Romanche n'ont pas èté étudiés.

Cette rivière, après sa confluence avec le torrent du Lautaret; coule parmi des schistes et quelques blocs cristallins. Elle reçoit 
de nombreux affluents du massif de la Meije. Dáns la Combe de Malaval, la Romanche traverse à nouveau des terrains cristallins et, à $1040 \mathrm{~m}$, débouche dans le lac Chambon.

Le 4-IX-1967, le cours d'eau était très pollue après le Frêneyd'Oisans $(930 \mathrm{~m})$, ̀̀ tel point que toutes les pierres émergées étaient recouvertes d'un épais enduit visqueux et brunâtre. La pollution devait être récente, car de nombreuses nymphes de Blepharoceridae s'observaient sur les parties émergées des blocs. En amont de Bourgd'Oisans, la Romanche s'approfondit et sa pente devient très faible; elle reçoit un affluent très important. Le Vénéon. En aval de Bourgd'Oisans, les eaux sont de nouveau polluées et le restent pendant la traversée des gorges. Entre Séchilienne et Vizille, elles s'épurent, car des animaux aquatiques peuvent être récoltés. Les déversements au niveau de cette dernière ville appauvrissent à nouveau considérablement la faune de la Romanche, qui se jette dans le Drac en aval de Montchaboud. La pollution augmente encore jusqu'à Grenoble.

Le 4-IX-1967, la source du Rif du Col était assez importante et une femelle de $W$. beckeri y a été capturée. L'eau se perdait à environ 50 mètres de là parmi les cailloutis du lit. Le torrent du Lautaret réapparaissait au bas de la pente, mais aucun Empidide n'a été recueilli avant la descente rapide du torrent sur Arsine. A la hauteur de ce hameau et vers $1670 \mathrm{~m}$, des $W$. aerea ont été capturées sur des blocs de calcschistes. Dans la Romanche, en amont de sa confluence avec le torrent du Lautaret et un peu en aval, à $1650 \mathrm{~m}$, de rares W. hygrobia pouvaient être observées. Les Empidides de la même espèce étaient beaucoup plus nombreux à Villar-d'Arène $(1545 \mathrm{~m})$, à la Grave ( $1450 \mathrm{~m}$ et $1440 \mathrm{~m}$ ) et à quelques mètres en amont du lac Chambon, à $1050 \mathrm{~m}$. Ils devenaient fort rares à $940 \mathrm{~m}$, avant le foyer de pollution du Frêney-d'Oisans. Aucun Empidide n'a été vu dans le lit de la Romanche en aval du village. Plus bas, au lieudit Le Clapier, en amont de Bourg-d'Oisans, les $W$. phantasma n'étaient pas rares et il $\mathbf{y}$ en avait aussi quelques-unes dans le Vénéon, à $2 \mathrm{~km}$ de la confluence. Entre Bourg-d'Oisans et Séchilienne, je n'ai pas réussi à apercevoir des Hemerodromiinae dans la Romanche, mais entre Vizille et Séchilienne, à $4 \mathbf{~ k m}$ de ce dernier village, vers $340 \mathrm{~m}$, ainsi qu'au Péage de Vizille, vers $310 \mathrm{~m}$, les $W$. fallaciosa étaient abondantes. Dans le Drac, pas plus que dans l'Isère en aval de Grenoble, je n'ai pu récolter d'Empidides, sans doute à cause de la pollution; mais dans cette dernière rivière, en amont de Grenoble, entre Saint-Ismier et Domène, les $W$. phantasma étaient nombreuses et il y avait aussi quelques $W$. fallaciosa. 
La Durance. - Cette rivière prend sa source dans des prairies humides du massif du Gondran, vers 1860 mètres. Le 5-VIII-1967, la portion amont du cours d'eau était à sec jusqu'au village de Montgenèvre. Le lit recevait là des eaux usées, parfaitement azoïques. Mais, plus bas, des affluents amenaient une augmeniation du débit et, en face du hameau des Alberts, à 1430 mètres d'altitude, une riche population torrenticole était installée sur les blocs cristallins du cours d'eau, qui descendait rapidement. A Prelles, la Durance coule en pente faible sur un lit de sable et de graviers, qui est remplacé à Saint-Clément surtout par des schistes. A Embrun, la Durance s'écoule dans la vaste retenue de Serre-Ponçon $(800 \mathrm{~m})$ puis elle s'étale et son cours devient relativement lent.

Aucune Wiedemannia n'a été capturée le 5-VIII-1967 au-dessus des eaux putrides à Montgenèvre, mais à 1430 mètres d'altitude, les $W$. aquilex et surtout les $W$. hygrobia étaient extrêmement abondantes. A Prelles (1 $130 \mathrm{~m}$ ), elles étaient remplacées par des $W$. fallaciosa. A Saint-Clément $(877 \mathrm{~m})$, la même espèce était représentée, mais accompagnée de $W$. phantasma. Je n'ai fait aucune autre récolte sur la Durance, si ce n'est le 14-VIII-1959 un peu en amont de Sisteron et vers $500 \mathrm{~m}$ d'altitude; les $W$. bistigma étaient extrêmement abondantes.

Le Guil, peu avant sa confluence avec la Durance, vers $1000 \mathrm{~m}$, n'avait que des $W$. fallaciosa.

Le Bruyant et le Furon. - Le Furon est un cours d'eau à débit très irrégulier du Massif du Vercors, sa faune a été étudiée le 8-IX-1967.

Il prend naissance dans des prairies humides de la « Combe Claire », vers $1400 \mathrm{~m}$ d'altitude. Dans sa partie amont il est intermittent et, le 8 septembre 1967, il était à sec jusqu'au voisinage du hameau de Furon, à $1140 \mathrm{~m}$ d'altitude; il sortait d'un étang et coulait sous forme d'un ruisselet. A la hauteur de Lans-en-Vercors, son débit était plus important. Entre l'Écluse et l'Olette, plusieurs sources ferrugineuses se déversent dans le Furon. Plus loin, la pente diminue et le cours devient très lent; de grandes touffes de Potamogeton occupent les parties latérales du ruissé̃u. Ayant dépassé l'Olette, le Furon s'engage dans les gorges d'Engins; sa pente s'accentue légèrement en restant régulière; des Mousses remplacent les Potamogeton. Le Furon reçoit, sur sa gauche, un important affluent, le Bruyant. Un peu en amont du village d'Engins, situé à $837 \mathrm{~m}$, il traverse un étang de pisciculture, dévale rapidement, à découvert, jusqu'à $300 \mathrm{~m}$; les touffes de Mousses sont alors peu nombreuses et de petite taille. Il s'engage ensuite dans les gorges de Sassenage, très boisées, et coule sur des dalles calcaires ou parmi de 
gros blocs recouverts de Mousses (Platyhypnidium rusciforme); il $y$ : reçoit un affluent froid et assez permanent, sorti d'une grotte, les "Cuves de Sassenage ». Le faciès du Furon change brusquement vers $210 \mathrm{~m}$, avant la traversée de Sassenage; il devient un cours d'eau de plaine, lent et pollué, qui ne tarde pas à se jeter dans l'Isère.

Le Bruyant, principal affluent du Furon, naît d'une source rhéocrène vers $1000 \mathrm{~m}$ d'altitude et coule dans une gorge encaissée entre de hautes falaises calcaires; le débit est assez régulier et la température de l'eau peu variable, surtout dans la portion amont $\mathrm{du}$ ruisseau. La pente est faible et les touffes de Mousses sont fort étendues. Le Bruyant se jette dans le Furon à $894 \mathrm{~m}$ d'altitude.

Une seule femelle de Wiedemannia fallaciosa a pu être capturée en amont de l'Olette, à $974 \mathrm{~m}$ d'altitude et, un peu plus bas que ce hameau, de nombreux Empididae de la même espèce pouvaient s'observer sur les pierres. Dans les gorges d'Engins, une récolte a été effectuée en amont du débouché du Bruyant, à $900 \mathrm{~m}$ d'altitude; elle était formée, en quantités presque égales, de $W$. fallaciosa et de W. oxystoma dolichocephala; au-delà du débouché du Bruyant, dans la Combe de l'Ours, à $867 \mathrm{~m}$, les $W$. fallaciosa étaient abondantes, mais les $W$. oxystoma dolichocephala rares. Dans la partie raide du Furon, en-dessous du hameau de l'Eglise et à $420 \mathrm{~m}$, seules les $W$. fallaciosa étaient représentées. Au-dessus de Sassenage, dans la partie ombragée du torrent, à $240 \mathrm{~m}$, les $W$. boheniani étaient extrênement nombreuses, mais je n'ai pas réussi à trouver des exemplaires d'autres espèces; le 28-VI-1903, J. Villeneuve a trouvé, dans cette même portion du Furon, des W. oxystoma (certainement de la sous-espèce dolichocephala), ainsi que des $W$. hygrobia. En aval de Sassenage et à $208 \mathrm{~m}$, j'ai capturé uniquement des W. fallaciosa.

Quant au Bruyant, il n'avait pas d'Empididae près de sa source; à une centaine de mètres de cel'e-ci apparaissaient les $W$. bohemani et plus loin les $W$. ouedorum; à un kilomètre et demi de la source, dans une partie plus ensoleillée, les W. rhynchops-alpina et les $W$. aquilex se mêlaient aux Diptères des espèces précédentes.

La Vence. - Ce ruisseau, au débit très irrégulier, prend naissance dans le massif de la Chartreuse sur les pentes de Chamechaude, vers $2100 \mathrm{~m}$ d'altitude (Source du Pré Boiteux), mais, le 7-IX-1967, le ruisseau était à sec jusqu'à la hauteur du hameau Le Churut, vers $1099 \mathrm{~m}$, et devenait ensuite un mince ruisselet fort pollué. Un cours d'eau de même importance le rejoignait au Molard-Giroud, à $960 \mathrm{~m}$. Après la traversée de la route du Sappey à Grenoble, la pente de la Vence s'accentue, des sources augmentent son débit et diminuent la pollution, puis le ruisseau s'engage dans un vallon et reste très ombragé jusqu'à sa confluence, à $470 \mathrm{~m}$, avec le ruisseau de Sarcenas, 
à peu près aussi important que lui. La Vence coule ensuite dans une gorge, sur des poudingues à ciment calcaire puis traverse l'important village de la Monta à $260 \mathrm{~m}$; il y devient très pollué et presque azoïque, puis se jette dans l'Isère.

Aucun Empidide n'a été trouvé dans la Vence avant sa sortie du hameau de Pillonnières à $920 \mathrm{~m}$; les $W$. fallaciosa étaient alors abondantes. Dans la partie ombragée, à $480 \mathrm{~m}$, seuls les $W$. ouedorum étaient représentées et, à $610 \mathrm{~m}$, en amont de la confluence avec le ruisseau de Sarcenas, elles cohabitaient avec des $W$. fallaciosa. A $480 \mathrm{~m}$, en aval de la confluence, les $W$. fallaciosa étaient seules à nouveau et elles l'étaient encore à $265 \mathrm{~m}$, avant que la Vence ne pénètre dans La Monta. Le village traversé, le ruisseau n'avait plus d'Empidides.

La Loire. - Elle naît à $1550 \mathrm{~m}$ au Gerbier de Jonc, parmi des débris phonolithiques et des basaltes; la source, rhéocrène et à débit régulier, forme un ruisselet qui traverse un étang encombré de végétation et riche en débris humiques, de sorte que la Loire a très vite une population importante. A 1500 mètres, le 13-VIII-1963, des $W$. rhynchops arvernensis et des W. bohemani cohabitaient; à Rieutord $\left(\begin{array}{ll}1 & 123 \mathrm{~m}\end{array}\right)$ j'ai retrouvé des exemplaires de la première espèce, mais mêlés à des $W$. zetterstedti. L'étude de cette rivière n'a malheureusement pas été poursuivie plus loin.

\section{DISCUSSION}

Dans le tableau page 287, je donne une liste des Wiedemannia des Pyrénées, du Massif Central et des Alpes, avec leurs limites altitudinales, telles qu'on les connaît actuellement. Dans une même colonne horizontale, la même espèce figure deux ou trois fois, ou bien, à défaut, plusieurs sous-espèces sont indiquėes, ou encore deux ou trois espèces affines; celles qui n'ont pas encore été signalées de France et pour lesquelles je possède peu de documents sont mises entre parenthèses.

Nous voyons qu'aucun Wiedemannia du Massif Central ne dépasse $1300 \mathrm{~m}$, ce qui n'a rien de surprenant, étant donné que peu de cours d'eau y sont assez importants, au-dessus de cette limite altitudinale. Le Massif Central possède trois espèces, représentées aussi dans les Pyrénées et les Alpes, $W$. bohemani, $W$. ouedorum et $W$. bistigma, une forme commune avec les Pyrénées, W. rhynchops mirousei, une espèce qui figure aussi dans les Alpes orientales, $W$. lamellata et une espèce, $W$. zetterstedti, représentée également en Europe Centrale; enfin une espèce, $W$. bifida et la sous-espèce $W$. rhynchops arvernensis seraient endémiques du Massif Central. La plupart de ces 
Wiedemannia se trouvent aussi bien dans le bassin de la Garonne que dans celui du Rhône et sans doute également dans celui de la Loire, formant parfois les mêmes associations. C'est ainsi que, dans le Lot à Nojaret, en amont de Badaroux et vers $800 \mathrm{~m}$. le 10-VIII-1967, ainsi que dans la Loire à Rieutord, à $1123 \mathrm{~m}$, le 13-VIII-1963, j'ai capturé à la fois des $W$. rhynchops arvernensis et des $W$. zetterstedti. L'association tripartite $W$. bistigma, W. bifida et W. zetterstedti a été observée dans le Tarn, entre Pont du Tarn $(540 \mathrm{~m})$ et Sainte-Enimie $(470 \mathrm{~m})$ en août 1967 , comme dans l'Eyrieux à SaintSauveur de Montagut $(217 \mathrm{~m})$, le 12-VII-1962. W. bohemani se trouve dans le Tarn à $875 \mathrm{~m}$, dans les affluents de l'Eyrieux, près de Marcols-les-Eaux, entre 700 et $900 \mathrm{~m}$ d'altitude, et enfin dans la Loire, près de sa source, à $1500 \mathrm{~m}$. Le Massif Central formerait donc un ensemble homogène, en ce qui concerne les Dipteres faisant l'objet de cette étude.

Pour les Alpes et les Pyrénées, il y a lieu de distinguer d'une part les espèces dont l'aire est restreinte aux parties hautes, comme $W$. beckeri, et d'autre part celles n'habitant que les régions basses, comme $W$. bistigma, ou qui ont une vaste répartition verticale, comme $W$. hygrobia.

Les premières ont toutes leur territoire limité, en France, à l'une ou à l'autre des deux régions montagneuses et sc seraient formées par isolement géographique ${ }^{3}$, à partir d'une souche commune, de sorte qu'à chaque espèce pyrénéenne correspond une espèce, ou tout au moins une sous-espèce différente dans les Alpes. C'est le cas pour $W$. angelieri, $W$. oredonensis et $W$. beckeri pyrenaica, dont les homologues alpins sont respectivement $W$. queyrasiana, $W$. longicornis et $W$. beckeri alpina.

Quant aux espèces de la seconde catégorie, elles se seraient dispersées le long des cours d'eau de faible altitude, profitant des fluctuations de leurs aires écologiques, amenées par des modifications du climat et $\mathrm{du}$ réseau hydrographique. Toutefois, quatre sousespèces de $W$. rhynchops, et $W$. falcifera ainsi que $W$. languedocica ont des affinités respectivement avec deux espèces alpines.

Il y a lieu de s'étonner des étranges discordances dans la répartition altitudinale de certaines espèces de Wiedemannia :

$W$. ouedorum, en Afrique du Nord, se rencontre jusqu'à $3000 \mathrm{~m}$ d'altitude, mais il est très commun dans les oueds des régions basses; dans l'île de Majorque, on le trouve jusqu'au niveau de la mer dans des eaux fort tièdes. Or, dans les Pyrénées comme dans le Massif Central, cette même espèce semble avoir une répartition altitudinale très courte et s'accomoder d'eaux froides seulement. C'est

3. Le Massif Central étant trop peu élevé pour servir de relai. 
ainsi qu'elle est abondante dans plusieurs ruisseaux des environs d'Aubrac (Lozère) entre 1100 et $1300 \mathrm{~m}$ d'altitude.

W. fallaciosa ne se trouve, dans le Grand Atlas Marocain, que dans les parties hautes, entre 1740 et $3250 \mathrm{~m}$; il en serait presque de même dans les Pyrénées, où cette espèce paraît rare. Dans les Alpes, $W$. fallaciosa est l'espèce la plus commune et on la trouve seule dans beaucoup de petits cours d'eau entre 80 et $1000 \mathrm{~m}$, aussi bien que dans de grandes rivières. Peul-être ces Empidides entrent-ils en compétition, sinon à l'état larvaire, tout au moins une fois devenus imagos, avec des Wiedemannia d'autres espèces,

Peut-on employer les Wiedemannia comme indicateurs de zones écologiques? Ils présentent pour celà un avantage sur d'autres animaux du domaine lotique. Il ne semble pas, en effet, qu'il y ait de relations entre la répartition des différentes espèces de ce genre et la nature du substrat. Ainsi des imagos de $W$. ouedorum, de $W$. fallaciosa et de $W$. bohemani ont été trouvées aussi bien sur des blocs granitiques, sur des basaltes que sur des calcaires, et les imagos de presque toutes les autres espèces se capturent sur des roches cristallines comme sur des roches sédimentaires riches en calcium. Toutefois des $W$. beckeri n'ont été observées jusqu'ici que sur des granits, mais cela peut être dû à ce que je n'ai jamais exp'oré des cours d'eaux très froids dans des massifs non cristallins; d'ailleurs j'ai réussi fort bien à élever des larves de cette espèce dans de la mousse provenant de tufs, avec de l'eau riche en calcium.

Cette indifférence vis-à-vis de la nature du substrat est sans doute due au fait que les Wiedemannia sont zoophages à l'état larvaire comme à l'état imaginal et se nourrissent de Chironomidae appartenant à n'importe quelle espèce.

Toutefois, les mêmes indicateurs ne peuvent être utilisés que dans une même région montagneuse. C'est ainsi que, dans les Pyrénées, $W$. ouedorum caractérise les petits ruisseaux de haute montagne, alors que, dans les Alpes, les Diptères de la même espèce, trop euryoques, sont de fort mauvais indicateurs, leur présence dans un torrent n'ayant aucune valeur significative, si ce n'est celle de montrer que l'eau n'est pas trop polluée.

Les larves des Wiedemannia présentent un autre avantage, celui d'être indifférentes vis-à-vis de la vitesse du courant, au-dessus d'une limite inférieure, qui est à peu près la même pour toutes les espèces. Ils sont en effet rhéobiontes et vivent dans des niches écologiques protégées du courant.

Les Turbellariés ont les mêmes avantages que les Wiedemannia, et pour des raisons analogues; malheureusement les espèces du domaine lotique sont peu nombreuses. 
Par contre les larves de beaucoup de Trichoptères et de Diptères Chironomidae, Psychodidae et Simuliidae ont des préférences à l'égard de la nature de la roche qui les porte, ou bien vis-à-vis de la vitesse du courant, et conviennent mal pour une étude de ce genre ${ }^{4}$.

J'ai partagé les espèces de Wiedemannia en cinq groupes, indiqués sur le tableau. Grâce à eux, nous pouvons distinguer sept zones dans un même cours d'eau; elles ne correspondent pas à celles de J. Illies et de L. Botosaneanu, qui ont basé leur classification sur des critères différents.

La zone 0 , dont je ne précise pas l'altitude, est dépourvue de Wiedemannia, mais héberge quelquefois des Atalanta ${ }^{5}$; elle comprend des sources et certains ruisselets de sources et de glaciers, dont les pierres ont une fort maigre couverture d'Algues, mais parfois des touffes de Bryophytes très étendues. lls ont peu ou pas du tout de larves de Chironomidae.

La zone A est caractérisée par les Wiedemannia du groupe A ne vivant pas en-dessous de 1500 mètres. Elle comprend les ruisselets de source de haute montagne, dont l'eau reste toujours froide (endessous de $10^{\circ} \mathrm{C}$ ), et qui sont sans couverture de neige pendant une courte période de l'année seulement; les pierres ont, au cours de la belle saison, un revêtement d'Algues important et, par conséquent, une riche population animale.

Zone $\mathrm{O}$ et $\mathrm{A}$, dans les Alpes, n'ont pas d'autre Triclade que Crenobia alpina et les truites n'y pénètrent généralement pas.

La zone B, dans laquelle vivent les Wiedemannia du groupe B, comprend des sections de ruisselets et de ruisseaux d'importance fort diverses et qui appartiennent à trois catégories. Celles de la première, situées au-dessus de $1500 \mathrm{~m}$, sont exposées au midi et ont une longue période de déneigement. Celles de la seconde sont en moyenne altitude, sur des versants moins bien exposés; celles de la troisième sont dans des régions basses, mais, pour des raisons diverses, la température de leur eau varie entre des limites très étroites et ne dépasse jamais $15^{\circ} \mathrm{C}$. Les Wiedemannia du groupe $\mathrm{B}$ ont une vaste répartition verticale et se trouvent tous occasionnellement au-dessus de $1500 \mathrm{~m}^{6}$. La zone B est, dans les Alpes, l'habitat à la fois de Crenobia alpina et de Polycelis felina.

4. J'ai essayé d'utiliser des Dolichopodidae en même' temps que des Wiedemannia. Ce sont également des Diptères zoophages, mais leurs larves sont soit ripicoles, soit madicoles et vivent par conséquent en marge de l'habitat lotique. Aussi leur étude n'a-t-elle fourni jusqu'à présent que des résultats peu concluants.

5. Atalanta est un genre voisin de Wiedemannia.

6. W. aquilex, espèce assez rare, se trouvera sans doute aussi au-dessus de $1500 \mathrm{~m}$. 
完

瓷

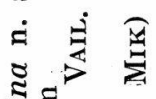

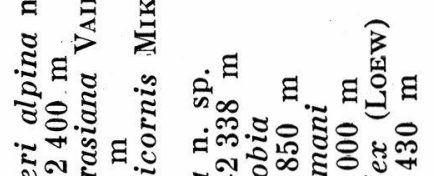

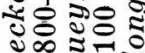

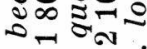

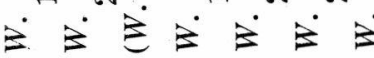

\section{¿}

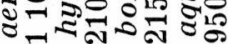
บิ

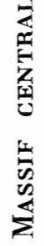
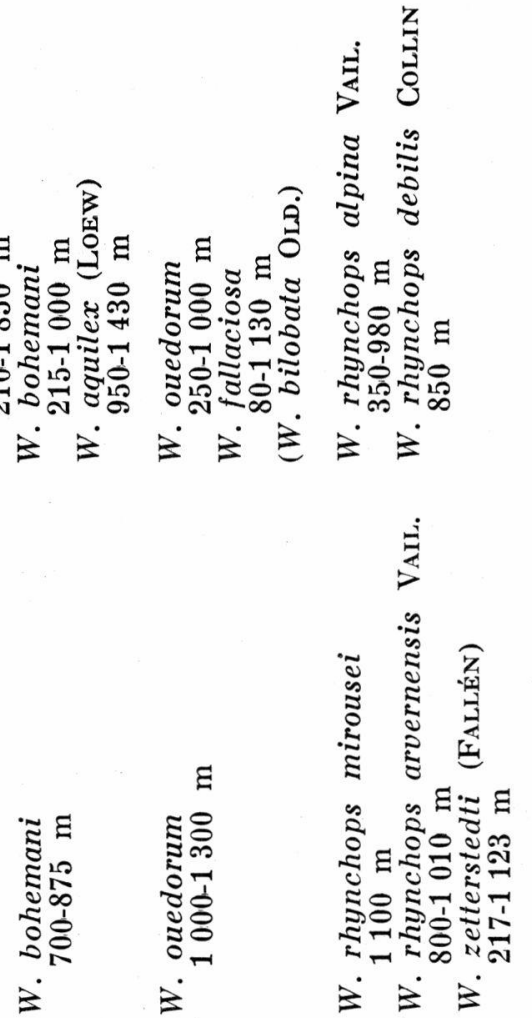

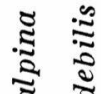

รี่

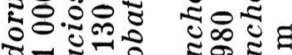

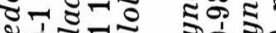

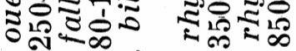

$\dot{3} \dot{E} \dot{E}$

$\dot{\hat{n}}$

鱼

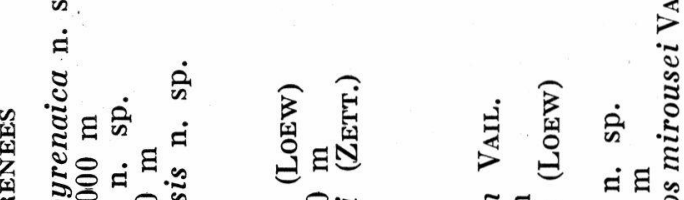

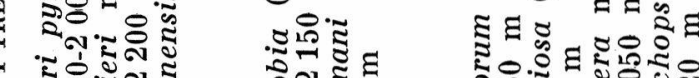

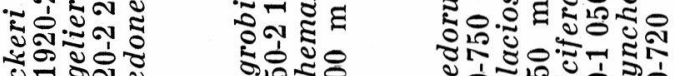

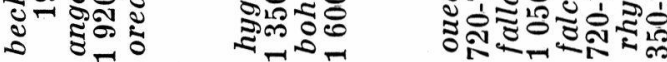

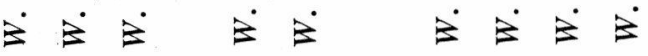

4

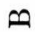

帘

㸃 $\mho$

嘧

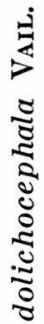

承

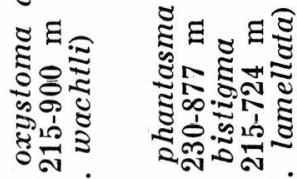

$\dot{B} \dot{B} E$
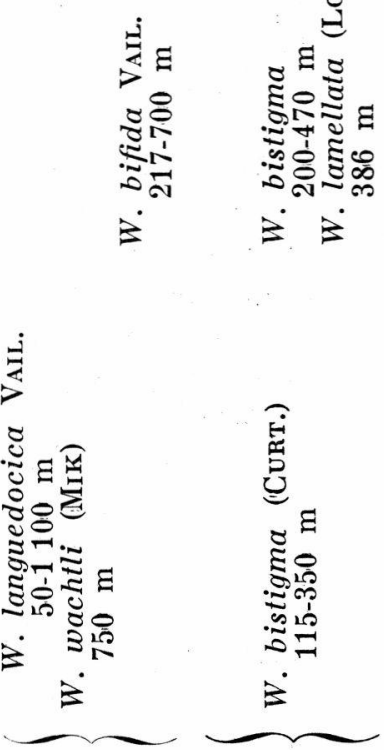

ค

되

我

资 
La zone $\mathrm{C}$ comprend des sections de ruisseaux et de rivières rapides, de moyenne et de basse altitude, dont la température de l'eau dépasse toujours $15^{\circ} \mathrm{C}$ en été, mais n'est pas très variable. Les Wiedemannia du groupe $\mathrm{C}$ se rencontrent parfois au-dessus de 1000 mètres, mais ne dépassent guère cette limite. Cette zone est l'habitat de Polycelis felina seule.

La zone D comprend les portions de cours d'eau de faible et de moyenne importance, de température très variable et de régions basses. Polycelis felina s'y rencontre avec Dendrocoelum lacteum. Les Wiedemannia du groupe $D$ ne s'observent pas au-dessus de $1000 \mathrm{~m}$.

La zone E, à Dendrocoelum lacteum, à Dugesia gonocephala, et parfois aussi à Polycelis felina, est l'ensemble des sections de rivières et de fleuves de plaine, à pente encore suffisante pour qu'il y ait un léger clapotis sur les pierres des berges. Elle recouvre seulement en partie la «Barbenregion» mais déborde sur elle vers l'amont. Les Wiedemannia du groupe $\mathrm{E}$ ont généralement une vaste répartition en Europe, en-dessous de $500 \mathrm{~m}$ d'altitude.

La zone $F$, dépourvue de Wiedemannia comme la zone $O$, comprend les parties lentes et calmes des cours d'eau de toute importance et à toutes les altitudes. Dans les régions basses, $\mathbf{F}$ représente les portions aval de la «Barbenregion» et se trouve souvent peuplée de Polycelis nigra.

La même espèce peut appartenir à deux groupes différents, suivant la région montagneuse à laquelle on a affaire. C'est ainsi que, dans le Massif Central, $W$. ouedorum, ainsi que $W$. rhynchops arvernensis, appartiendraient plutôt au groupe B qu'au groupe C et qu'inversement il conviendrait mieux de placer $W$. bohemani dans le groupe $C$ que dans le groupe $B$.

La zonation que je propose ici ne tient compte de l'altitude et de l'importance du cours d'eau que dans une certaine mesure; d'autres critères sont utilisés en même temps, notamment les variations annuelles de la température de l'eau, ainsi que la richesse globale de la population animale.

En effet, l'abondance des Wiedemannia est liée directement à celle des larves de Chironomidae; cette dernière est en relation étroite avec l'épaisseur de la couverture d'Algues et souvent avec la présence d'autres Insectes zoophages, qui se nourrissent de ces larves (Rhyacophila, Dicranota...). De nombreuses Wiedemannia, sur les pierres d'un cours d'eau rapide, indiquent généralement que sa population animale est riche dans son ensemble.

Parfois les seules Wiedemannia que l'on trouve dans une rivière sont de mauvais indicateurs de zones écologiques. Ainsi la Vence, en septembre 1967, n'hébergeait que des $W$. ouedorum et des $W$. fallaciosa. En pareil cas, on ne peut tirer aucune conclusion. 
Il est bien évident que l'absence de Wiedemannia ne prouve pas toujours qu'on ait affaire à la zone $\mathrm{O}$ ou à la zone $\mathrm{F}$. Elle peut être due à la pollution chimique ou organique de l'eau. D'autre part les parties d'un ruisseau qui s'assèchent en été peuvent rester dépourvues, parfois de façon régulière, d'Empididae de ce genre.

$2500 \mathrm{~m}$

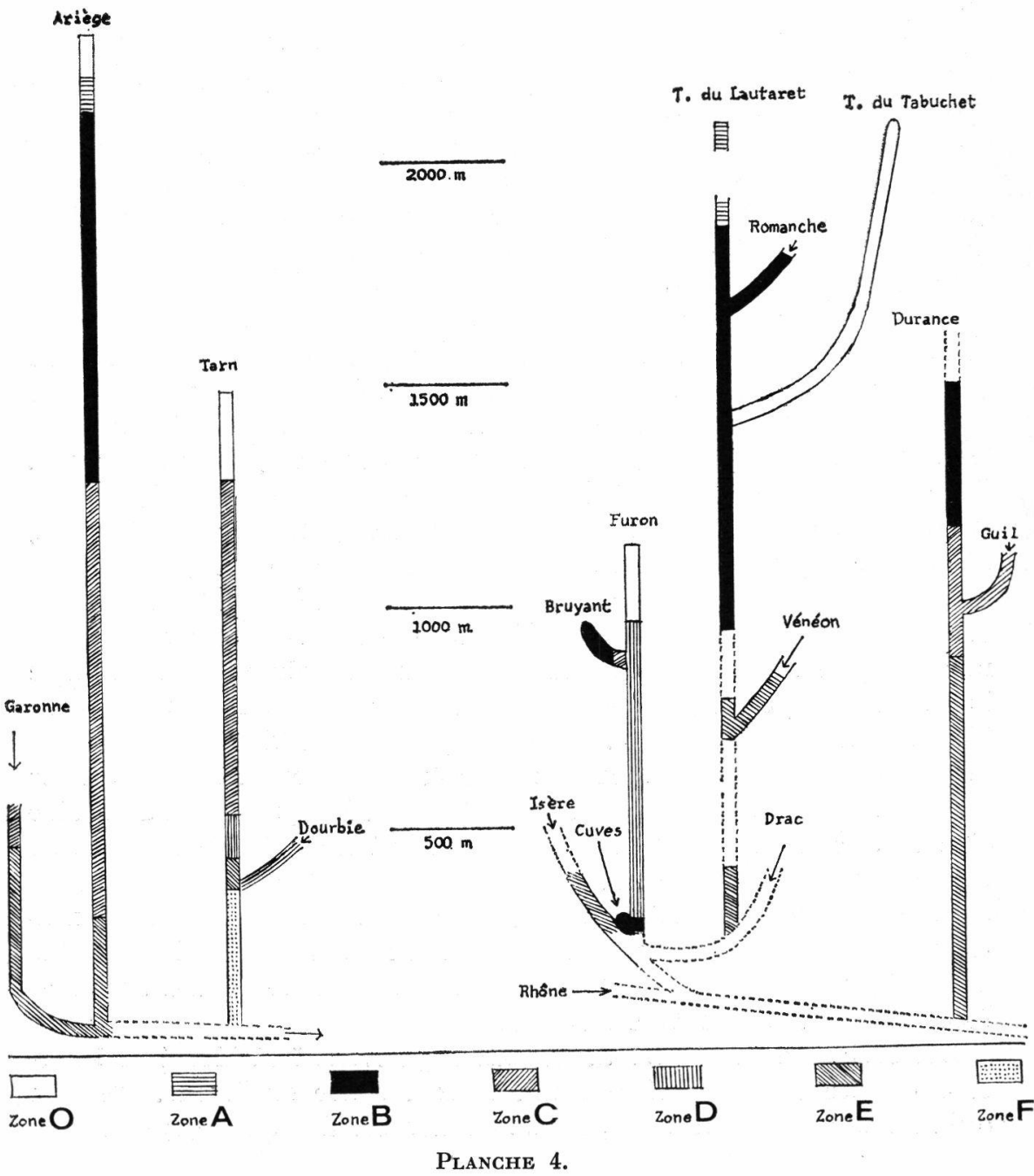

Représentation schématique des cours d'eau étudiés. Les portions dépourvues de Wiedemannia, sans doute à cause de la pollution, ont leurs contours figurés en pointillé. 
Il arrive que des espèces de deux ou même de trois groupes successifs se trouvent réunies dans la même portion d'un cours d'eau. Il faut alors ne tenir compte que de celles représentées par de nombreux sujets. Prenons un exemple : Le 9-VII-1964, j'ai capturé à $1100 \mathrm{~m}$ d'altitude, dans une portion fort ombragée du torrent Le Glandon, à Saint-Colomban-les-Villards (Savoie), un seul mâle de $W$. aerea, accompagné de nombreux $W$. hygrobia et de quelques $W$. fallaciosa. Il s'agissait évidemment d'une portion de la zone B, $W$. aerea et $W$. fallaciosa s'y trouvant près d'une des limites de leur territoire.

Bien entendu, certaines zones peuvent faire défaut dans un cours d'eau, ou être morcelées. C'est ainsi que les ruisseaux du Massif Central sont tous dépöurvus de zone $A$ et que leur zone $O$ se trouve directement raccordée soit à la zone $\mathrm{B}$, soit plus fréquemment à la zone $\mathrm{C}$; ce dernier cas est celui du Tarn, qui présente cependant de rares $W$. bohemani, à $875 \mathrm{~m}$, dans une portion très ombragée. Beaucoup de ruisseaux du Massif Central ont un important secteur $\mathrm{O}$, alors que la Loire, par suite de conditions spéciales, n'en a pas et débute dans la zone B. Dans les Alpes, le torrent du Lautaret (voir p. 279) n'a pas du tout de secteur $O$ et, au débouché de la source, les pierres du lit ont un épais revêtement de Diatomées ou d'Hydrures, avec une multitude de larves de Chironomidae; aussi les Wiedemannia s'y observent-elles par milliers en juillet et en août. Par contre, le torrent froid du Tabuchet, qui descent des glaciers de la Meije et se jette dans la Romanche au pied de La Grave, à $1445 \mathrm{~m}$, n'a pas de Wiedemannia et appartient en entier à la zone 0 .

On assiste parfois, en suivant le lit d'un cours d'eau de l'amont vers l'aval, à une succession de territoires en sens inverse de l'ordre normal. C'est ainsi qu'en descendant le Furon (voir p. 281), on passe brusquement, peu avant Sassenage, de la zone $D$ dans la zone B, en trouvant de nombreux $W$. bohemani, et même des $W$. hygrobia. Ce « retour en arrière » insolite est certainement dû à ce que le Furon, en cet endroit, reçoit d'importantes résurgences d'eau froide et à ce que les «Cuves de Sassenage » lui apportent en outre, de façon régulière, des eaux venues du domaine souterrain ${ }^{7}$. Cet exemple montre bien que deux portions de cours d'eau, situées à des altitudes très différentes, peuvent avoir des caractères écologiques beaucoup plus proches que deux portions localisées à des altitudes fort voisines et à la même latitude.

Des séries de territoires de la zone $F$ peuvent fort bien être intercalées entre des territoires d'une zone d'ordre supérieur. C'est ce । qui se produit pour le Tarn, dans sa traversée des Causses; quelques

7. En période de basses eaux, le ruisseau sorti des * Cuves de Sassenage * est bien plus important que le Furon, avant la confluence. 
longs segments de zone $F^{8}$ y alternent, tout d'abord avec de courts tronçons de zone $D$, puis avec des segments de zone $E$.

Il nous faut, pour terminer, aborder la question des confluences, dont J. Illies, dans son étude faunistique de la Fulda, a su si bien montrer l'importance. J'ai, comme lui, observé des modifications brutales de la faune au niveau de confluences, mais jamais dans le cours d'eau principal et par contre souvent dans l'affluent, lorsqu'il avait un débit beaucoup moins important que celui du premier. En utilisant les Wiedemannia comme zonateurs écologiques, j'ai fréquemment constaté qu'en un point de confluence, le cours d'eau principal appartenait à une zone d'ordre supérieur à celle de laquelle faisait partie son affluent. C'est ainsi que la Dourbie, juste avant qu'elle ne se jette dans le Tarn, appartient à la zone $\mathrm{D}$, ou même à la zone $\mathrm{C}$, alors que le Tarn fait lui-même partie de la zone $\mathrm{E}$; un autre exemple est celui du Bruyant, qui est du domaine de $C$, tandis que le Furon est de celui de E, juste avant leur confluence. Par contre, lorsque deux ruisseaux ou deux rivières fusionnent, elles conservent presque toujours, en aval de leur lieu de rencontre, les mêmes espèces de Wiedemannia.

Ceci pourrait laisser croire que le débit d'un segment de cours d'eau a une importance capitale pour lui assigner une valeur du point de vue écologique. En réalité, à altitude égale, les rivières et leurs affluents ne subissent pas les mêmes variations de température, lesquelles sont déterminées à la fois par l'importance du débit et par les changements climatiques des régions déjà traversées. Le critère de zonation le plus important ne serait-il pas celui des températures extrêmes?

Le présent travail n'est pas plus qu'une ébauche; il est destiné seulement a montrer l'intérêt des Wiedemannia pour une zonation écologique. Dans les cours d'eau étudiés, je n'ai mesuré la température de l'eau qu'au moment des captures, et par conséquent à des périodes de l'année et à des heures différentes; de tels relevés sont fort insuffisants. Pour une étude rigoureuse, il serait nécessaire d'effectuer d'une part des récoltes dans des stations plus rapprochées et plusieurs fois au cours d'une même année, et d'autre part des relevés de températures maxima et minima pour l'eau de chaque station.

\section{RÉSUMÉ}

Les Wiedemannia accompagnent presque partout les eaux à cours rapide. Leur absence est toujours significative, soit de pollution, soit d'une très grande pauvreté de la faune dans son ensemble.

8. Non figuré page 289 , dans un but de clarté. 
Sept zones - indiquées par des Wiedemannia de différentes espèces peuvent être distinguées, de l'amont vers l'aval, dans un même cours d'eau. En homologuant les territoires d'une même zone dans des rivières différentes, la zonation fournie par les Wiedemannia tient compte des températures extrêmes au cours de l'année et de l'abondance des animaux.

Trois sous-espèces de $W$. beckeri et quatre espèces sont décrites.

\section{THE DISTRIBUTION OF WIEDEMANNIA IN WATER COURSES AND THEIR USE AS INDICATORS OF ECOLOGICAL AREAS.}

There are, in France, 23 species of Wiedemannia; all live, as larvae and pupae, in brooks, streams and rivers; after they have emerged, they stay near by on stones partly overflowed by water. Many species can be distributed along a same water course, according to the elevation, to the flow, to the abundance of the aquatic fauna as a whole, and especially to the maximal and minimal temperature of the water through the year. They seem to be indifferent to the kind of rock they lay on and eat Chironomids of one species or another.

As several species have close ecological requirements and others have different ones, the author tries to use the Wiedemannia for a zonation.

Seven zones are chosen, two of them without any Wiedemannia.

Three subspecies of $W$. beckeri and four new species are described.

\section{DIE VERBREITUNG DER WIEDEMANNIA IN DEN WASSERIAAUFER UND IHR WERT ZUM KENNZFICHNEN VON ÖKOLOGISCHEN ZONEN.}

Die Wiedemannia kommen fast überall in schnellfliessenden Gewässern vor. Ihre Abwesenheit weist immer entweder auf Verunreinigung oder auf das geringe Vorhandensein der Tierwelt in ihrer Gesamtheit hin.

Man kann sieben Zonen, die von verschiedenartigen Wiedemannia bezeichnet sind, in demselben Fluss von Gebirg zu Tal unterscheiden. Wenn man die Gebiete einer und derselben Zone in verschiedenen Flüssen registriert, stellt man fest, dass die durch die Beobachtung der Wiedemannia vorgenommene Zoneneinteilung im Verhältnis zu den Maximal- und Minimaltemperaturen im Verlauf des Jahres und zur grossen Anzahl der Tiere steht.

Drei Unterarten von $W$. beckeri und vier neue Arten werden hier beschrieben.

\section{TRAVAUX CITÉS}

BährmanN (R.). 1960. - Vergleichend-morphologische Untersuchungen der männlichen Kopulationsorgane bei Empididen. Beitr. z. Entomol., 10 : 485-540.

Engel (O.). 1938-1940. - in E. Lindner, II « Die Fliegen der Palaearktischen Region 》, 4, Stuttgart.

ILlies (J.). 1953. - Die Besiedlung der Fulda, nach dem jetzigen Stand der Untersuchung. Ber. Limnol. Flussstat. Freudenthal, 5 : 1-28.

ILliEs (J.). 1961. - Versuch einer allgemeinen biozönotischen Gliederung der Fliessgewässer. Internat. Rev. ges. Hydrobiol., 46 : 205-213. 
Illies (J.) et Botosaneanu (L.). 1963. - Problèmes et méthodes de la classification et de la zonation écologique des eaux courantes, considérées surtout du point de vue faunistique. Mitt. int. Ver. Limnol., 12 : 1-57.

ILliES (J.). 1967. - Limnofauna Europaea, Stuttgart.

Vaillant (F.). 1951. - Un Empidide destructeur de Simulies. Bull. Soc. zool. Fr., '7\% : 371-379.

Vaillant (F.). 1956. - Contribution à l'étude des Diptères Empididae du Grand Atlas marocain. Bull. Soc. Sc. nat. phys. Maroc, 36 : 61-71.

Vaillant (F.). 1956. - Quelques Wiedemannia de France nouveaux ou peu connus (Diptera, Empididae). L'Entomologiste, 12 : 11-16.

Vaillant (F.). 1964. - Révision des Empididae Hemerodromiinae de France, d'Espagne et d'Afrique du Nord (Dipt.). Ann. Soc. ent. Fr., 133 : 143-171.

Villeneuve (J.). 1905. - Coup d'œil sur la Faune diptér )logique des Alpes françaises. Congrès de l'A.F.A.S., Grenoble, 33 : 883-890.

\author{
(Laboratoire de Zoologie, \\ Faculté des Sciences, \\ 38-Saint-Martin-d'Hères.)
}

